\title{
$\checkmark$ Research Square \\ Genetic testing for the diagnosis and classification of joint hypermobility: a case report of 15 patients
}

\section{Lanlan Zeng}

Central South University School of Life Sciences

\section{Li Zhang}

Central South University School of Life Sciences

\section{Siyuan Linpeng}

Central South University School of Life Sciences

\section{Zhuo Li}

Central South University School of Life Sciences

\section{Desheng Liang}

Central South University School of Life Sciences

lingqian Wu ( $\square$ wulingqian@sklmg.edu.cn )

Central South University School of Life Sciences https://orcid.org/0000-0002-7070-5896

\section{Research}

Keywords: Ehlers Danlos syndrome, joint hypermobility, whole-exome sequencing, CHST14

Posted Date: February 18th, 2020

DOl: https://doi.org/10.21203/rs.2.23844/v1

License: (a) (1) This work is licensed under a Creative Commons Attribution 4.0 International License. Read Full License 


\section{Abstract}

\section{Background}

Joint hypermobility $(\mathrm{JH})$ is used to define the capability of a joint moving passively or actively beyond normal limits along physiological axes, which can be influenced by multiple factors (genetic factors, age, gender, weight and training), and the accurate incidence is unclear. In this study, we aimed to identify the genetic cause of $\mathrm{JH}$ in 15 patients from seven unrelated Chinese families.

Results

We identified seven pathogenic/likely-pathogenic variants: two novel mutations, in the COL6A2 and CHST14 genes, and five reported mutations in the COL11A1, NALCN , GALNS and COL5A1 respectively. Based on the genetic testing, we were able to diagnose the precise condition for each patient: Stickler syndrome in Proband 1, the Ullrich congenital muscular dystrophy in proband 2, CLIFAHDD syndrome in proband 3, Mucopolysaccharidosis IVA in proband 4, Classical Ehlers-Danlos syndrome (EDS) in proband 5, and Musculocontractural EDS in proband 6. Moreover, this is the first time to describe the Musculocontractural EDS caused by CHST14 in China. Though the expression of the mRNA and protein have not significantly changed, we speculated that the mutation of the CHST14 may affect the sulfotransterase activity of the protein.

\section{Conclusions}

The diagnosis in all probands except proband 6 and 7 were corrected following genetic analysis, indicating the importance of the genetic testing in the diagnosis and classification of $\mathrm{JH}$ cases. Our findings also offered insight into the genotype-phenotypes relationship and expanded the mutation spectrum of the disease-causing genes in $\mathrm{JH}$.

\section{Background}

Joint hypermobility $(\mathrm{JH})$ is a descriptor rather than a diagnosis, which is universally accepted to define the capability that a joint (or a group of joints) could move beyond normal limits along physiological axes, passively or actively[1]. JH may exist as an isolated diagnostic finding or a feature of a larger syndromic diagnosis[1], which mainly seen in hereditary disorder of connective tissue, like Ehlers-Danlos syndrome (EDS), joint hypermobility syndrome (JHS) and related disorders. It is reported that $\mathrm{JH}$ can be influenced by multiple factors, such as genetic factors, age, gender, weight and training, and the exact incidence is unclear.

Ehlers-Danlos Syndrome (EDS) is a rare clinically and genetically heterogeneous connective tissue disorder (HCTDs), which mainly characterized by joint hypermobility, tissue fragility and skin hyperextensibility, the clinical spectrum varies from joint hypermobility to severe physical disability and life-threatening complications [2]. In 1998, the villefranche classification recognizes six subtypes based on the biochemical and molecular basis [3], with the advent of next-generation sequencing, many new genes have been identified, the international EDS Consortium revised EDS subtypes and recognized 13 subtypes [3]. More 
than 20 genes are reported to be associated with this syndrome, the clinical diagnosis of all the 13 EDS subtypes is based on a set of major and minor clinical criteria. Even based on this, it is still quite difficult to establish a definitive diagnosis because of its variable and overlap of clinical phenotypes. Emerging evidence shows that genetic analysis can provide precise diagnosis and genetic counselling.

Joint hypermobility syndrome (JHS) and the hypermobile type of Ehlers-Danlos syndrome (hEDS) are clinical diagnoses in the absence of other causation, which has been used to describe in those with musculoskeletal complaints including joint hypermobility, joint subluxations/dislocations[4]. JHS was first descripted by Kirk et al and Grahame et al[ $[5,6]$, the clinical spectrum was often clinically indistinguishable from hEDS[7], and the clinical diagnosis of JHS was based on Brighton tests and criteria[5]. Some experts found that JHS and hEDS may co-exist in the same pedigrees and could not be distinguished in the familial cases[8], so the current EDS nosology combines these two entities into the hypermobile type of EDS[4]. Though other patterns of inheritance, hEDS is often thought as an autosomal dominant disorder of connective tissue, and there is no known genetic etiology and accurate prevalence estimation studies of this disease[4]. In recently years, some genes (TNXB, COL3A1, LZTS1) variants have been found in the patients with hEDS[9-12]. With the wider use of whole-exome sequencing (WES), it expected that some additional hEDS-related genes will be identified.

In the study, we collected 15 patients with a clinical presentation of joint hypermobility from seven unrelated Chinese families. These patients had been initially diagnosed with suspected EDS and/or JHS, according to the published diagnostic criteria. Sanger sequencing, CNV sequencing and whole-exome sequencing were performed in all patients and seven pathogenic/likely-pathogenic variants were detected.

\section{Subjects And Methods}

\subsection{Subjects}

From 2008 to 2018, 15 patients (from 7 unrelated families), who presented joint hypermobility with or without other symptoms and were initially diagnosed with suspected Ehlers-Danlos syndrome or joint hypermobility syndrome (JHS), were recruited at the Hunan JiaHui Genetic Hospital. Informed consent was obtained from the patients' families. This study was approved by the Ethics Board of the State Key Laboratory of Medical Genetics of China, Central South University (approval number: 2016102101).

Proband 1: Proband 1 was a 25-year-old woman, born to nonconsanguineous parents. She was found that her two legs were different in lengths at the first years old, diagnosed with hips dislocation at the age of 6 years old, and then treated with surgery. She can walk normally but couldn't squat when she came to our hospital, no other phenotypic abnormality was discovered. She gave birth to a daughter who also presented hip dislocation after birth. We diagnosed them as suspected joint hypermobility syndrome when she came to our hospital at the year of 2008.

Proband 2: Proband 2 was a seven-year-old boy, who was the first children of nonconsanguineous parents. He started walk slowly with abnormal gait (wadding gait) at the first years old, and diagnosed as congenital dislocation of hip at the XiangYa hospital, then he was performed manual reduction. On examination at the 
age of 7 years, in addition to his previous symptoms, he had difficulty in lifting shoulders and stretching straightly his elbow joints. Besides these, he also had dysstasia, muscle hypotonia and bottle-shaped caries. We diagnosed him as suspected EDS when he came to our hospital at the year of 2011.

Proband 3: Proband 3 was a five-year-old girl, who was born with deformed hands (first phalanx was long, flexion contractures of proximal interphalangeal joints) (Fig. 1.B), and presented with dislocation of the hips and underwent conservative treatment at the two months old. The proband also presented with delayed psychomotor development (sit alone at the one year and six months old. She can only speak some simple word, and cannot walk alone at present). Magnetic resonance imaging (MRI) results showed cerebral atrophy. Metabolic screening and chromosome karyotyping were normal. No other phenotypic abnormality was found. After rehabilitation therapy at the local hospital, her symptoms have improved. We diagnosed her as $\mathrm{JH}$-related disorder according to her symptoms.

Proband 4: The proband 4 was three-year-old, the big sister of the twins, who were diagnosed with rickets at birth and treated with calcium supplements at local hospital. Her symptoms gradually worsened due to irregular medication. The proband presented with joint hypermobility of the wrist (flexion activity was $180^{\circ}$ ), pectus carinatum, coccyx protruding and eversion of inferior costal border when came to our hospital. The ALP was $230 \mathrm{U} / \mathrm{L}$ (normal range: $118-390 \mathrm{U} / \mathrm{L}$ ), Ca $76.6 \mathrm{ug} / \mathrm{ml}$ (normal range: $54.0-81.6 \mathrm{ug} / \mathrm{ml}$ ), $\mathrm{P}$ $1.64 \mathrm{mmol} / \mathrm{L}$ (normal range: $1.45-2.1 \mathrm{mmol} / \mathrm{L}$ ), VitD3 $75.21 \mathrm{nmol} / \mathrm{L}$ (normal range: $75.0-175.0 \mathrm{nmol} / \mathrm{L}$ ) and BALP U/L (normal range: 0 - $U / L$. Three ossification centers can be seen in the left wrist joint $X$-ray, electroneurography was normal. Her twin sister has milder symptoms than her, and we diagnosed them as $\mathrm{JH}$-related disorder.

Proband 5: Proband 5 was a 28-year-old man, who presented with joint hypermobility at birth, and his fingers could be dorsiflexed close to forearms (Fig. 1.C1-C2), the joint space was large, and there was a sense of friction when moving, but the weight-bearing was not significantly affected. There were no vascular and joint malformations. The Ca was $46.07 \mathrm{mg} / \mathrm{L}$ (normal range: 54.0-81.6 $\propto \mathrm{g} / \mathrm{ml}$ ), X-ray showed that variation of distal ulnas was positive and the left side was obvious, suggesting that there might be impingement syndrome. He gave birth to a daughter who was found joint hypermobility after birth, and presented with dislocation of hip at the six months old, and then underwent surgery treatment due to the ineffective of conservative treatment. There were multiple patients presented with joint hypermobility, hip dislocation and slender fingers in this family, and we diagnosed them as suspected EDS.

Proband 6: Proband 6 was a four-year-old boy, the first child of a healthy consanguineous parents, who was born by cesarean section for premature rupture of fetal membranes at 38 weeks gestation. Postnatally he was found to have flexion contractures of proximal interphalangeal joints, adducted thumb and joint hypermobility of the wrist (Fig. 1.D1-D2). He was under treatment for development delay in the local hospital at the age of 7 months, and presented with mild scoliosis, delayed wounding healing and atrophic scarring when he was referred to our hospital. His mother aborted a fetus whose ultrasound results suggested talipes equinovarus and hands deformities later. According to the clinical symptoms, we suspected him as Musculocontractural EDS.

Proband 7: Proband 7 was a 11-year-old boy, born to a healthy nonconsanguineous parents, who presented with joint hypermobility and scoliosis after birth and scoliosis is severer with age (Fig. 1.E1-E3). He had 
surgery for cryptorchidism at the age of 3 years. On clinical examination at the age of 8 years old, he presented with marked generalized joint hypermobility, hypotonia, pectus carinatum and craniofacial dysmorphism (broad nasal bridge, blue sclera, bulbous nose, multiple nevi), he also had some atrophic scarring on his arms and legs. On examination at the age of 11 years, in addition to his previous symptoms, he had an eyeball rupture when his classmate bumped the head accidentally into his eyes. In addition, His parents once gave birth to a girl and a twin boy, they all presented with joint hypermobility and died for unknown reason after birth. According to the clinical symptoms, we diagnosed him as progeroid-type EDS.

\subsection{Methods}

Sangers sequencing or next-generation sequencing was used to identify their molecular etiology. Aiming at verifying the pathogenicity of the variant which was found in proband 6 , expression vectors was constructed, qualitative analysis and western blot were used to determine the CHST14 mRNA levels and protein levels, respectively.

\subsubsection{Mutation Analysis By Direct Sequencing}

Genomic DNA was extracted from the peripheral blood cells of all the subjects. As proband 6 was clinically suspected diagnosis as Musculocontractural EDS, we sequenced all the exon and intron-exon boundaries of CHST14. Proband 7 was clinically suspected diagnosis as progeroid-type EDS (Spondylodysplastic EDS), which mainly caused by B4GALT7 or B3GALT6, so we sequenced all the exon and intron-exon boundaries of the two genes.

\subsubsection{Copy Number Variants Sequencing}

Proband 4 and proband 7 were present multiple system abnormalities, so we also conducted copy number variants sequencing of them. $50 \mathrm{ng}$ of genomic DNA was fragmented to average size of $300 \mathrm{bp}$, and sequencing libraries were prepared as previously described[13]. Libraries were sequenced using the Hiseq2000 platform (Illumina Inc.) to generated approximately 8 million 36-bp single-end reads, representing 0.1 -fold genome coverage. All the sequences were aligned to the unmasked hg19 genome using the Burrows-wheeler algorithm. The theoretical log2 value for a duplication is log2 [1.5] $=0.58$ and for a deletion is $\log 2[0.5]=-1.0$. Cutoff copy number values used to call duplications were set at $>2.8(\log 2$ $[1.4]=0.49)$, and those used to call deletions were set at $<1.2(\log 2[0.6]=-0.74)$.

\subsubsection{Exome Capture And Sequencing}

Since 2017, whole-exome sequencing (WES) has been implemented for all our patients, including proband 6 who was identified an uncertain significance variant (VUS) of CHST14 by direct Sanger sequencing. The samples underwent WES according to the manufacturer's protocols. The exomes were captured using the xGen ${ }^{\circledR}$ Exome Research Panel v1.0 (Integrated DNA Technologies) and sequenced on an Illumina 
Hiseq2000 (Illumina, San Diego, CA, USA) with 100-bp paired-end reads. We analyzed all the variants that meeting all of the following requirements: 1 . Minor allele frequency (MAF) $<5 \%$ according to the 1000 Genomes Project, ESP6500 and the Exome Aggregation Consortium (ExAC) (http://exac.broadinstitute.org/) database; 2. Variants in introns and synonymous mutations were filtered out; 3 . Predicted to be damaging by SIFT, PolyPhen_2, Mutation Taster and Human splicing Finder. Candidate variants were then confirmed by Sanger sequencing.

\subsubsection{Construction Of Expression Vectors}

The human wild-type CHST14 (complementary DNA, cDNA) was donated by the Han Lab of Xiamen University, the mammalian expression vector PCMV-N-HA containing an N-terminal HA tag (Beyotime, shanghai, China) was used for plasmid construction. To introduce the mutation into the WT-CHST14, the Mut Express II Fast Mutagenesis Kit V2(Vazyme Biotech, China) was used according to the manufacturer's protocol. Site-directed mutagenesis was performed with the primers, $5^{\prime}$ -

CCGGAATTCCGGTCACTGCTGACACGCCTCCTTGGTG-3' and 5'-

CCGCTCGAGCGGATGTTCCCCCGCCCGCTGACC-3'. The constructs were confirmed by sanger sequencing.

\subsubsection{Cell Culture And Transfection}

Human embryonic kidney (HEK) 293 cells were cultured in Dulbecco's modified Eagle's medium (DMEM), which supplemented with 10\% fetal calf serum (FCS). Wild-type (WT-CHST14), mutant-CHST14 (C362F) and empty-expressing vector with an HA tag were transiently transfected into HEK 293 cells by using Lipofectamine ${ }^{\circledR} 2000$ Transfection Reagent (Invitrogen) according to the manufacturer's protocol, respectively.

\subsubsection{Real-time Quantitative PCR}

Total RNA was extracted from patients' immortalized lymphoblast and cultured HEK 293 cells after 48 hours transfection using Trizol reagent (Invitrogen, Carlsbad, CA, USA) in accordance with standard procedures, and was reverse transcribed to the first strand cDNA with $1 \mu \mathrm{g}$ of the extracted RNA in a final volume of $20 \mu \mathrm{l}$ using RevertAid First Strand cDNA Synthesis Kit (Thermo scientific). Quantitative PCR was performed on a real-time PCR system (step one plus, ABI, USA) using an EvaGreen 2X qPCR MasterMix -ROX (abm) following the cycling conditions: $95^{\circ} \mathrm{C}$ for $10 \mathrm{~min}$ followed by 40 cycles of $95^{\circ} \mathrm{C}$ for $10 \mathrm{~s}$, and $60{ }^{\circ} \mathrm{C}$ for $1 \mathrm{~min}$. The relative standard curve method was used to analyze the expression level. The expression was normalized to Actin in the same sample and three biological repeats were measured. The real-time primer pairs for CHST14 were 5'-CCAAGGTGGCCTGCTCTAA-3' and 5'- TCACTGCGGTGGTCCATCTT - 3' (product length: $97 \mathrm{bp}$ ); The real-time primer pairs for actin were 5'-CGTCTTCCCCTCCATCGT-3' and 5'GAAGGTGTGGTGCCAGATTT - 3' (product length: 184 bp). 


\subsubsection{Western Blot Analysis}

HEK 293 cells were washed and extracted with 0.1\% Sodium Dodecyl Sulfate (SDS) buffer (Sigma) containing protease inhibitor cocktail (Sigma) after forty-eight hours transfection, and then the proteins were quantitated using the BCA Protein Assay Kit (Thermo Scientific). $20 \mu \mathrm{g}$ of total protein was subjected to $10 \%$ SDS-PAGE and transferred to a polyvinylidene difluoride (PVDF) membrane after electrophoretic. HAtagged proteins were detected with mouse anti-HA antibody (1:1000 dilution; Beyotime).

\subsubsection{Bioinformatics Analysis Of The Mutations}

The possible effects of the mutations on the function and structure of protein, and likelihood of pathological damage were analyzed by tools including SIFT, Mutation taster, Polyphen-2 and Mutation Assessor.

Using the protein structure modeling tool Phyre2, we predicted the structure of the wild-type (Fig. 2.G1-G3) and mutant for the lumenal domain (residues 61-376) of CHST14 (Fig. 2.H1-H3). The structures prediction was performed using the maltose binding protein-heparan sulfate 6-o- (template:c5t0aB).

\section{Results}

The genetic findings for all the probands have been listed in Table 1. 
Table 1

Summary of molecular findings in our probands

\begin{tabular}{|c|c|c|c|c|c|c|c|}
\hline $\begin{array}{l}\text { Proband } \\
\text { ID }\end{array}$ & Gene & $\begin{array}{l}\text { Mutation } \\
\text { at } \\
\text { nucleotide } \\
\text { level }\end{array}$ & $\begin{array}{l}\text { Mutation } \\
\text { at } \\
\text { protein } \\
\text { level }\end{array}$ & $\begin{array}{l}\text { Mutation } \\
\text { type }\end{array}$ & Segregation & ACMG & Reference \\
\hline 1 & COL11A1 & c. $560 \mathrm{C}>\mathrm{T}$ & T187M & Missense & NPS & Pathogenic & [27] \\
\hline 2 & COL6A2 & $\begin{array}{l}\text { c. } 954+ \\
1 G>A\end{array}$ & - & $\begin{array}{l}\text { Splice- } \\
\text { site }\end{array}$ & NPS & Pathogenic & - \\
\hline 3 & NALCN & $\begin{array}{l}\text { c. } 3542 G> \\
\text { A }\end{array}$ & R1181Q & Missense & De novo & Pathogenic & {$[24,28]$} \\
\hline 4 & GALNS & $\begin{array}{l}\text { c. } 1156 \mathrm{C}> \\
\mathrm{T} ; \\
\text { C. } 1097 \mathrm{~T}> \\
\mathrm{C}\end{array}$ & $\begin{array}{l}\text { R386C; } \\
\text { L366P }\end{array}$ & Missense & Het, M, F & Pathogenic & [29-32] \\
\hline 5 & COL5A1 & $\begin{array}{l}\text { c. } 655-2 A \\
>G\end{array}$ & - & $\begin{array}{l}\text { Splice- } \\
\text { site }\end{array}$ & NPS & Pathogenic & [33] \\
\hline 6 & CHST14 & $\begin{array}{l}\mathrm{c} .1085 \mathrm{~T}> \\
\mathrm{G}\end{array}$ & F362C & Missense & Het, M, F & VUS & - \\
\hline 7 & - & - & - & - & - & - & - \\
\hline
\end{tabular}

\subsection{Sequencing And CNV Results}

Direct bidirectional sequencing revealed that proband 6 was homozygous for the (c.1085T > G; p.F362C) missense mutation in CHST14, which was not found in the 100 normal controls, and both of his parents were heterozygous carriers of the mutation (Fig. 2.A). The mutation was also not reported in the Human Gene Mutation Database (HGMD) or ExAC, and it was likely to be disease-causing according to the prediction software, but it was interpreted as VUS (PM2, PP3, PP4) according to the American College of Medical Genetics and Genomics (ACMG) and Association for Molecular Pathology (AMP) variantInterpretation guidelines. Proband 7 didn't find any suspected pathogenic variants in B4GALT7 and B3GALT6. In order to identify whether there were some CNV variants in proband 3 and proband 7 , we conducted CNV sequencing of them, but failed to find any suspected pathogenic variants.

\subsection{WES Results}

WES sequencing revealed that proband 1 was a reported heterozygous missense mutation (c.560C $>T$ ) in COL11A1 gene. A novel heterozygous splicing mutation (c.954 + 1G >A) of COL6A2 was found in proband 2, which was judged to be pathogenic (PVS1, PM2, PP3) according to ACMG-AMP guidelines. A reported heterozygous missense mutation (c.3542G $>$ A) of NALCN was found in proband 3 . Reported compound 
heterozygous missense mutations (c.1156C > T; c.1097T > C) of GALNS were found in proband 4 and her little sister, her parents were carriers of the two mutations respectively. A reported heterozygous splicing mutation (c.655-2A $>\mathrm{G}$ ) of COL5A1 was found in proband 5 and all patients in his family. Proband 6 was not found any suspected pathogenic variants, except the homozygous missense mutation of CHST14 (c.1085T > G). We also didn't find any suspected pathogenic variants in proband 7 .

\subsection{RT-qPCR And Western Blotting Results}

We performed RT-qPCR to compare the mRNA expression levels between proband 6 and normal control, and also compared it between the mutant-CHST14 (C362F) and WT-CHST14, but there were unaltered compared to the normal control/wild-type (Fig. 2.C). Then, we performed western blotting to compare protein level between the mutant-CHST14 (C362F) and WT-CHST14, but also didn't find any significant difference between them (Fig. 2.D).

\subsection{In Silico Functional Analysis}

To better understand the effect of the mutation on protein function, we analyzed the mutation in silico with SIFT, PolyPhen-2, Mutation Taster and Mutation Assessor. Based on this analysis, the mutations found in our study were predicted to be deleterious. The conservation analysis via PolyPhen-2 revealed that the mutation position (p.F362C) of CHST14 is highly conserved among different species (Fig. 2.B).

\section{Discussion}

\subsection{Functional analysis of a novel variant}

In this study, all the discovered variants were classified as pathogenic or likely-pathogenic, except for the missense mutation (c.1085C > T) in CHST14, which was classified as VUS. CHST14 encodes dermatan-4sulfotransferase-1(D4ST1), which catalyzes the 4-0-sulfation of N-acetylgalactosamine (GalNAc) residues in dermatan sulfate, and was previously reported to cause autosomal recessive musculocontractural EDS ( $m c E D S)$ in Online Mendelian Inheritance in Man database (OMIM). The dermatan-4-sulfotransferase- 1 is comprised of 376 residues, which contains cytoplasmic, transmembrane and lumenal domains (Fig. 2.G). 22 mutations of CHST14 have been listed in the Human gene mutation database (Professional 2019.4), $54.5 \%$ of them are missense/nonsense mutations, and all of them locate at the lumenal domain (Fig. 2.G). In order to investigate the pathogenicity of the mutation in our study, we constructed expression vectors of the CHST14, but didn't find any significant differences in protein and mRNA levels between the wild-type and mutant one, this was consistent with the previous reports of other CHST14 missense mutations whose sulfotransterase activity were decreased $[14,15]$. We failed to detect sulfotransterase activity of the mutation due to lack of the key substrate (Desulfated Dermatan Sulfate was not sold at present). The mutation (c.1085T > G; p.F362C) in our study also locates at the luminal domain. In addition, we found that the amino acid polarity was changed and the 3D structure of C362 (Fig. 2.H2) was different from the F362 (Fig. 2.G2), which suggesting the mutation could impair the conformation of the residue side chain. We also 
found that the F362 can form one H-bonds with Y358 and A366 respectively, while the C362 can form two $\mathrm{H}$-bonds with Y358 and one H-bonds with A366 (Fig. 2.G3, Fig. 2.H3), suggesting the mutation may change the stability of D4ST1, and then affect the activity of D4ST1. In a word, the symptoms of our patient highly meet the diagnostic criteria of mcEDS[3], and the inheritance pattern is also consistent with mcEDS, so our conclusion is that the mutation is "likely-pathogenic" for this proband.

\subsection{Case Which Needs Special Mention}

Proband 7 is worth of special mention. The boy presented with marked generalized joint hypermobility, scoliosis, cryptorchidism, hypotonia, pectus carinatum and craniofacial dysmorphism, he also had some atrophic scarring on his arms and legs. When firstly referred to our hospital, we diagnosed him as progeroidtype EDS according to the characteristic clinical features [18-20], which changed its name as spondylodysplastic EDS (spEDS) $[3,19,21]$, and caused by B4GALT7 or B3GALT6 gene. Then we conducted sanger sequencing of the two genes in him, but didn't find any pathogenic variants. During the follow-up, we found that his twin brother died shortly after birth, and the symptoms were similar to his sister. And then, we conducted WES and CNV of the family, but still didn't find any pathogenic variants. The clinical symptoms of the proband were consistent with the diagnosis of EDS, but we didn't find any obvious pathogenic mutations of the genes known to cause EDS. We speculate that there may be some other gene which can lead to Ehlers-Danlos syndrome, and this type of EDS is likely to cause embryo death. In addition, we also can't rule out the proband is caused by gross deletion of B4GALT7 or B3GALT6. We will continue to find the possible causes of the family.

\subsection{Correlation Between Phenotypes And Genotypes In The Patient}

At present, the clinical diagnosis of EDS and JHS mainly depends on the clinical symptoms and the major and minor clinical criteria. However, there are still some JH-related diseases, which can't get an accurate diagnosis according to the clinical symptoms. Thereof, it is very important for clinicians to recognize this disease through the few main manifestations and existing detection technology. All the patients in our study share the same main symptom - joint hypermobility, and were diagnosed with suspected EDS and/or JHS at first, we refreshed their clinical diagnosis after genetic analysis.

Proband 6 was diagnosed with mcEDS according to the clinical criteria, and the genetic results confirmed it. Proband 5 was diagnosed with EDS, and the genetic results refreshed it as classical EDS. In this study, only the preliminary diagnosis of proband 6 was consistent with the final diagnosis, and the main reason was that the characterized clinical features, such as typical facial appearance and congenital contractures of thumbs and feet, which can differentiate it from other EDS subtypes. Though the proband 5 was also diagnosed as EDS, we can't diagnose him as specific subtype according to their symptoms due to the overlapping of phenotypes among different EDS subtypes. 
Proband 2 was diagnosed with suspected EDS, but the genetic results revelated a splicing mutation of COL6A2, a known Ullrich congenital muscular dystrophy gene[22], which characterized by distal hypermobility, proximal joint contractures, protruding calcanei, scoliosis and respiratory insufficiency. The proband 2 in our study mainly presented with dislocation of hip and contractures of shoulders and elbow joints, which can't get an accurate diagnosis according to the clinical symptoms. Proband 1, proband 3 and proband 4 were diagnosed with suspected $\mathrm{JHS} / \mathrm{JH}$-related disorders, the genetic results revelated mutations in COL11A1, NALCN and GALNS, which can lead to Stickler syndrome, CLIFAHDD syndrome, Mucopolysaccharidosis IVA, respectively[16, 23-26]. The genetic results corrected the first clinical diagnosis. The reasons for the discrepancy between clinical preliminary diagnosis and final diagnosis mainly due to the overlapping of phenotypes among different diseases, which also indicated the high heterogeneity of $\mathrm{JH}$.

\section{Conclusion}

In this study, we identified seven variants in the COL6A2, CHST14, COL11A1, NALCN, GALNS and COL5A1 genes, including two novel mutations in COL6A2, CHST14, and five already reported mutations in COL11A1, NALCN, GALNS and COL5A1. All the identified variants were most likely responsible for the disease. These findings offered an important insight into the pathogenic mutation spectrum of the corresponding genes and may be helpful for the clinical diagnosis and genetic counseling of patients with $\mathrm{JH}$. Moreover, we described the Musculocontractural EDS caused by CHST14 in China at the first time.

\section{Declarations}

\section{Acknowledgments}

We deeply thank the patients' family for their helps and supports in this study.

\section{Authors' contributions}

Lanlan Zeng, Lingqian Wu and Desheng Liang collaborated to the conception of the work; Lanlan Zeng and Lingqian Wu collaborated to the design of the work;Lanlan Zeng and Li Zhang were responsible for acquisition, analysis, and interpretation of data; Lanlan Zeng drafted the work. Siyuan Linpeng, Zhuo Li and Lingqian Wu substantially revised the work.

\section{Funding}

This work was supported by the grants from the National Key R\&D Program of China (2018YFC1002201,2017YFC1001802), the Major Scientific and Technological Projects for Collaborative Prevention and Control of Birth Defects in Hunan Province区2019SK10108区and the National Natural Science Foundation of China (81771599, 81974240).

Data availability statement: The data that support the findings of this study are available from the corresponding author upon reasonable request. 


\section{Ethics approval and consent to participate}

All procedures followed were in accordance with the ethical standards of the responsible institutional committee on human experimentation and with Helsinki Declaration of 1975(revised in 2000). The study was approved by the Ethics Board of the State Key Laboratory of Medical Genetics of China, Central South University (2016102101)

\section{Consent for publication}

Written, informed consents were obtained from the patients' family.

Conflict of interest: There is no conflict of interest.

\section{Author details}

1, Center for Medical Genetics\& Hunan Key Laboratory of Medical Genetics, School of Life Sciences, Central South University, 110 Xiangya Road, Changsha, Hunan 410078, PR China

\section{References}

1. Castori, M., et al., A framework for the classification of joint hypermobility and related conditions. Am J Med Genet C Semin Med Genet, 2017. 175(1): p. 148-157.

2. Malfait, F. and A. De Paepe, The Ehlers-Danlos syndrome. Adv Exp Med Biol, 2014. 802: p. 129-43.

3. Malfait, F., et al., The 2017 international classification of the Ehlers-Danlos syndromes. Am J Med Genet C Semin Med Genet, 2017. 175(1): p. 8-26.

4. Tinkle, B., et al., Hypermobile Ehlers-Danlos syndrome (a.k.a. Ehlers-Danlos syndrome Type III and Ehlers-Danlos syndrome hypermobility type): Clinical description and natural history. Am J Med Genet C Semin Med Genet, 2017. 175(1): p. 48-69.

5. Grahame, R., H.A. Bird, and A. Child, The revised (Brighton 1998) criteria for the diagnosis of benign joint hypermobility syndrome (BJHS). J Rheumatol, 2000. 27(7): p. 1777-9.

6. Kirk, J.A., B.M. Ansell, and E.G. Bywaters, The hypermobility syndrome. Musculoskeletal complaints associated with generalized joint hypermobility. Ann Rheum Dis, 1967. 26(5): p. 419-25.

7. Tinkle, B.T., et al., The lack of clinical distinction between the hypermobility type of Ehlers-Danlos syndrome and the joint hypermobility syndrome (a.k.a. hypermobility syndrome). Am J Med Genet A, 2009. 149A(11): p. 2368-70.

8. Castori, M., et al., Nosology and inheritance pattern(s) of joint hypermobility syndrome and EhlersDanlos syndrome, hypermobility type: a study of intrafamilial and interfamilial variability in 23 Italian pedigrees. Am J Med Genet A, 2014. 164A(12): p. 3010-20.

9. Zweers, M.C., et al., Haploinsufficiency of TNXB is associated with hypermobility type of Ehlers-Danlos syndrome. Am J Hum Genet, 2003. 73(1): p. 214-7.

10. Morissette, R., et al., Broadening the Spectrum of Ehlers Danlos Syndrome in Patients With Congenital Adrenal Hyperplasia. J Clin Endocrinol Metab, 2015. 100(8): p. E1143-52. 
11. Narcisi, P., et al., A family with Ehlers-Danlos syndrome type III/articular hypermobility syndrome has a glycine 637 to serine substitution in type III collagen. Hum Mol Genet, 1994. 3(9): p. 1617-20.

12. Syx, D., et al., Ehlers-Danlos Syndrome, Hypermobility Type, Is Linked to Chromosome 8p22-8p21.1 in an Extended Belgian Family. Dis Markers, 2015. 2015: p. 828970.

13. Liang, D., et al., Non-invasive prenatal testing of fetal whole chromosome aneuploidy by massively parallel sequencing. Prenat Diagn, 2013. 33(5): p. 409-15.

14. Syx, D., et al., Genetic heterogeneity and clinical variability in musculocontractural Ehlers-Danlos syndrome caused by impaired dermatan sulfate biosynthesis. Hum Mutat, 2015. 36(5): p. 535-47.

15. Miyake, N., et al., Loss-of-function mutations of CHST14 in a new type of Ehlers-Danlos syndrome. Hum Mutat, 2010. 31(8): p. 966-74.

16. Patel, N., et al., GZF1 Mutations Expand the Genetic Heterogeneity of Larsen Syndrome. Am J Hum Genet, 2017. 100(5): p. 831-836.

17. Fukuda, N., et al., Identification of a novel glial cell line-derived neurotrophic factor-inducible gene required for renal branching morphogenesis. J Biol Chem, 2003. 278(50): p. 50386-92.

18. Salter, C.G., et al., Further defining the phenotypic spectrum of B4GALT7 mutations. Am J Med Genet A, 2016. 170(6): p. 1556-63.

19. Guo, M.H., et al., Redefining the progeroid form of Ehlers-Danlos syndrome: report of the fourth patient with B4GALT7 deficiency and review of the literature. Am J Med Genet A, 2013. 161A(10): p. 2519-27.

20. Hernandez, A., et al., A distinct variant of the Ehlers-Danlos syndrome. Clin Genet, 1979. 16(5): p. 335-9.

21. Ritelli, M., et al., Expanding the clinical and mutational spectrum of B4GALT7-spondylodysplastic Ehlers-Danlos syndrome. Orphanet J Rare Dis, 2017. 12(1): p. 153.

22. Yonekawa, T. and I. Nishino, Ullrich congenital muscular dystrophy: clinicopathological features, natural history and pathomechanism(s). J Neurol Neurosurg Psychiatry, 2015. 86(3): p. 280-7.

23. Acke, F.R., et al., Cephalometrics in Stickler syndrome: Objectification of the typical facial appearance. J Craniomaxillofac Surg, 2016. 44(7): p. 848-53.

24. Chong, J.X., et al., De novo mutations in NALCN cause a syndrome characterized by congenital contractures of the limbs and face, hypotonia, and developmental delay. Am J Hum Genet, 2015. 96(3): p. 462-73.

25. Peracha, H., et al., Molecular genetics and metabolism, special edition: Diagnosis, diagnosis and prognosis of Mucopolysaccharidosis IVA. Mol Genet Metab, 2018. 125(1-2): p. 18-37.

26. Khan, S., et al., Mucopolysaccharidosis IVA and glycosaminoglycans. Mol Genet Metab, 2017. 120(1-2): p. 78-95.

27. Miyagawa, M., et al., Targeted exon sequencing successfully discovers rare causative genes and clarifies the molecular epidemiology of Japanese deafness patients. PLoS One, 2013. 8(8): p. e71381.

28. Aoyagi, K., et al., A Gain-of-Function Mutation in NALCN in a Child with Intellectual Disability, Ataxia, and Arthrogryposis. Hum Mutat, 2015. 36(8): p. 753-7.

29. Guo, Y.B., et al., [Rapid prenatal genetic diagnosis of a fetus with a high risk for Morquio A syndrome]. Zhonghua Yi Xue Yi Chuan Xue Za Zhi, 2012. 29(2): p. 126-30. 
30. Ogawa, T., et al., Mucopolysaccharidosis IVA: screening and identification of mutations of the $\mathrm{N}$ acetylgalactosamine-6-sulfate sulfatase gene. Hum Mol Genet, 1995. 4(3): p. 341-9.

31. Fukuda, S., et al., Mucopolysaccharidosis IVA: submicroscopic deletion of 16q24.3 and a novel R386C mutation of $\mathrm{N}$-acetylgalactosamine-6-sulfate sulfatase gene in a classical Morquio disease. Hum Mutat, 1996. 7(2): p. 123-34.

32. Rivera-Colon, Y., et al., The structure of human GALNS reveals the molecular basis for mucopolysaccharidosis IV A. J Mol Biol, 2012. 423(5): p. 736-51.

33. Takahara, K., et al., Order of intron removal influences multiple splice outcomes, including a two-exon skip, in a COL5A1 acceptor-site mutation that results in abnormal pro-alpha1(V) N-propeptides and Ehlers-Danlos syndrome type I. Am J Hum Genet, 2002. 71(3): p. 451-65.

\section{Figures}


A
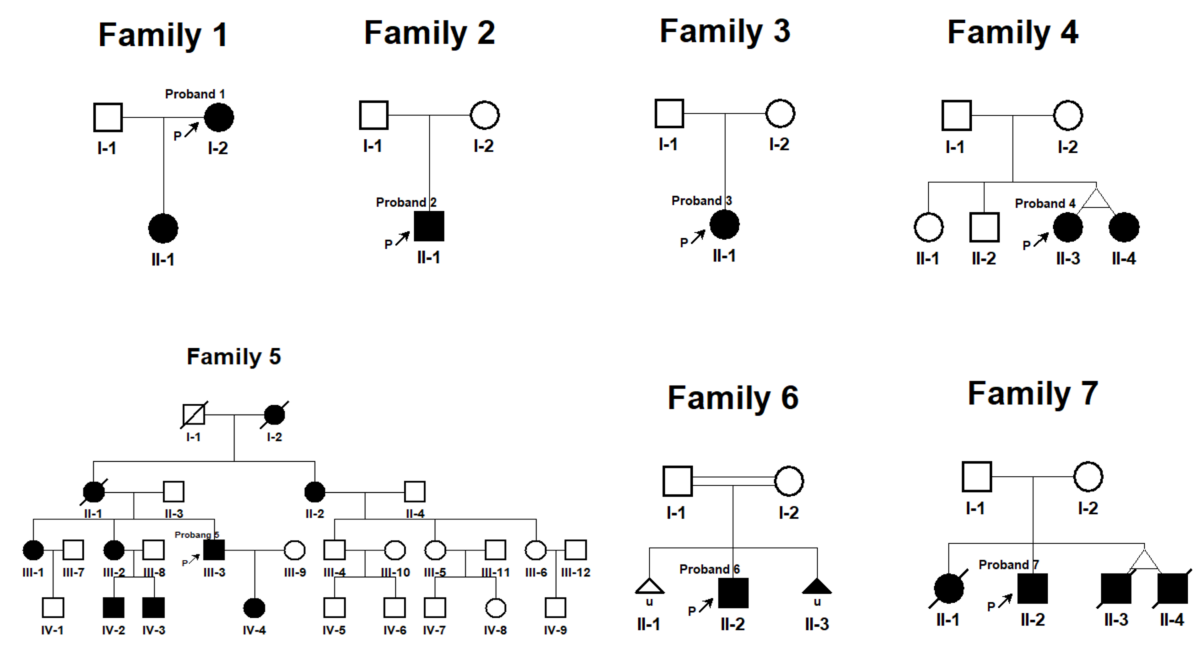

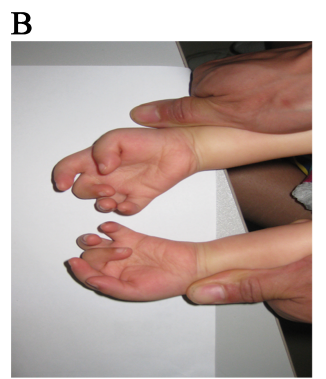

D1

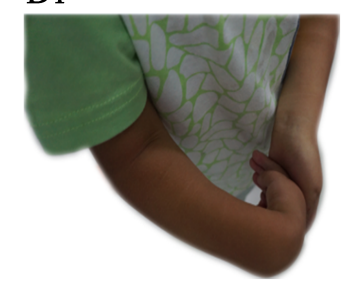

E2

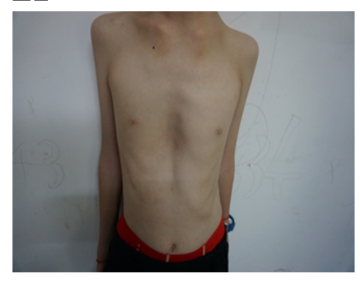

$\mathrm{C} 1$

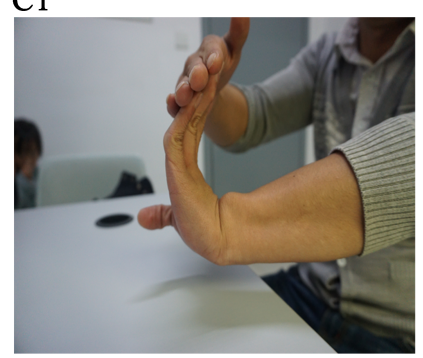

D2

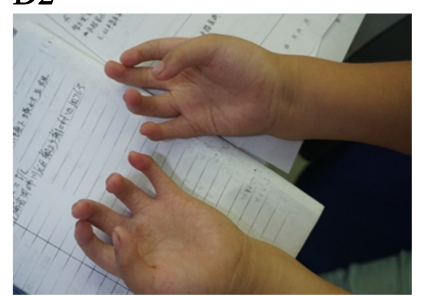

E3

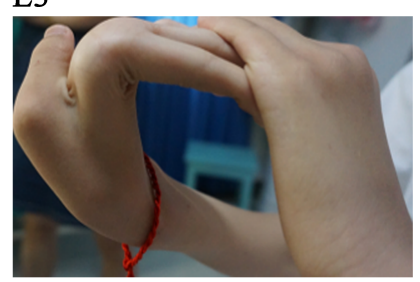

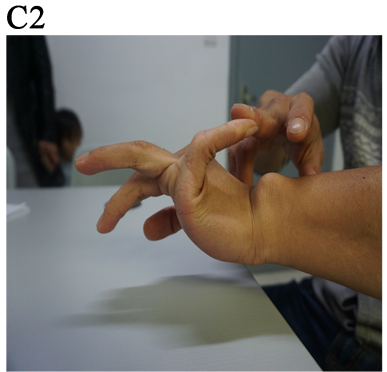

E1

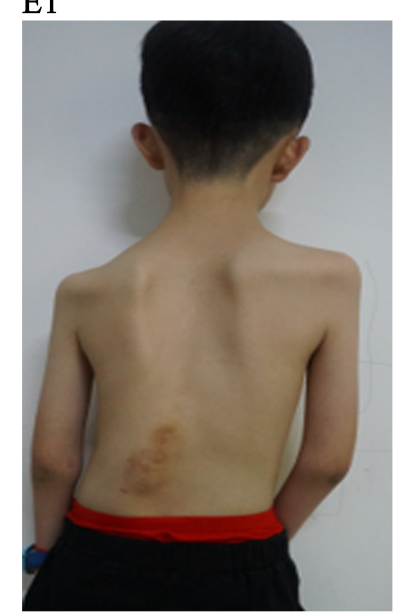

Figure 1

Clinical features and pedigrees of patients with joint hypermobility. A Pedigrees of patients with joint hypermobility. B Flexion contractures of proximal interphalangeal joints of proband 3. C1 -C2 Left hand shows joint hypermobility of proband 5. D1-D2: Clinical features of proband 6, D1 Joint hypermobility of the wrist; D2 Flexion contractures of proximal interphalangeal joints and adducted thumbs. E1-E3: Clinical features of proband 7, E1 scoliosis; E2 Pectus carinatum; E3 Joint laxity of the wrist and metacarpophalangeal joint. 

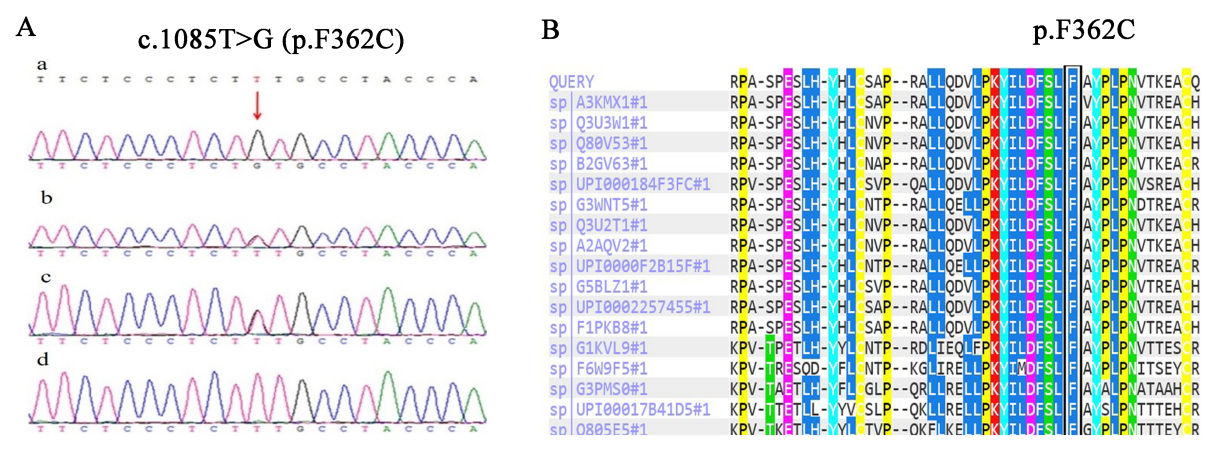

$\mathrm{C}$
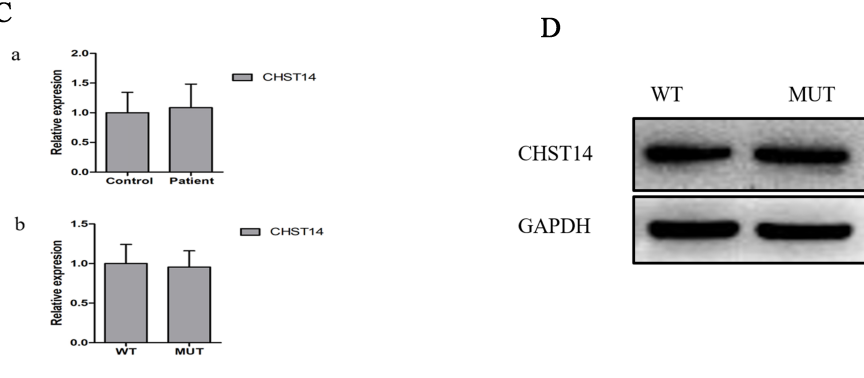

E
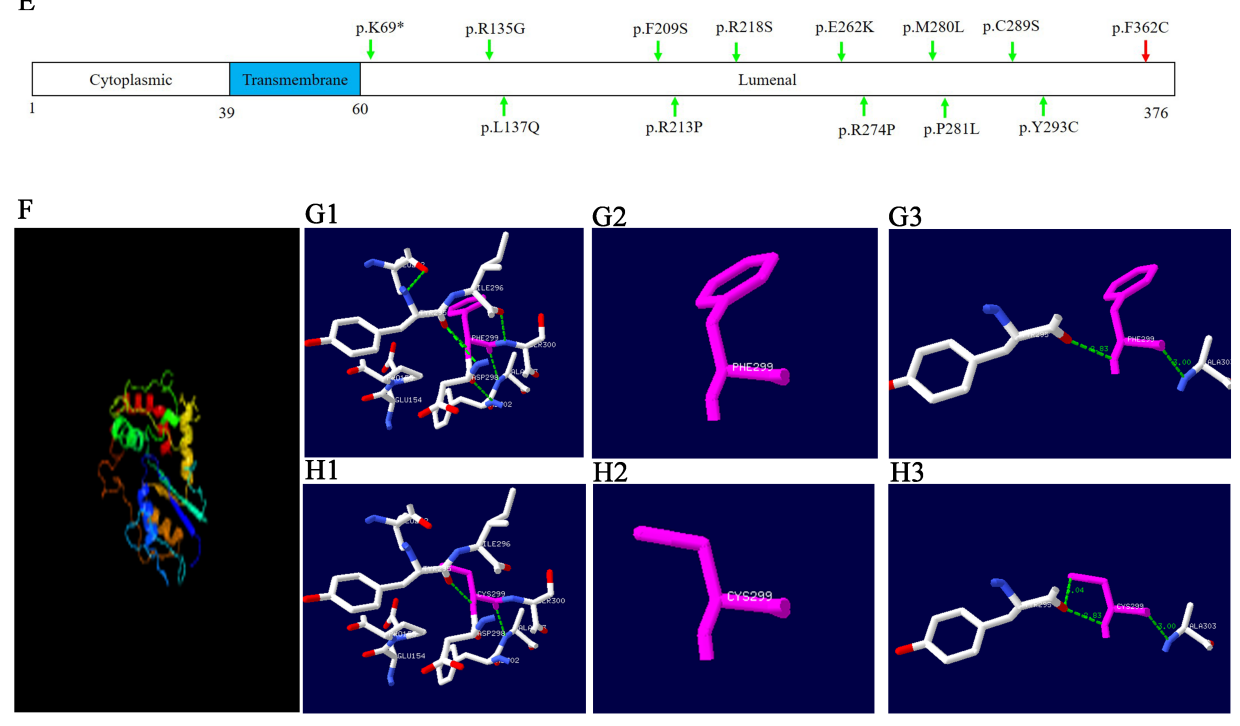

\section{Figure 2}

Genetic results of proband 6: A Sanger sequencing results of the proband $6(a)$, his parents $(b, c)$ and control(d), revealed a homozygous c.1085T>G substitution of CHST14 gene in the patient, his parents indicated heterozygous carriers. B Conservation analysis of CHST14 p. F362C. C Real time PCR results of the cell lines (a) and transfected HEK 293 cells (b). There is no significant difference between the control/WT and patient/mutant. D western blotting analysis of the WT and mutant CHST14. There is no significant difference between the WT and mutant CHST14. E The putative structural domains of CHST14, 
and variants identified is located in lumenal domain, the mutations reported were indicated by green arrows, while the mutation identified in our study was indicated by red arrow. F. Modeled structure of lumenal domain of the D4ST1 protein; G1-G3. Homology modeling of wild-type. G1. Neighboring residues of Phe362 in the wild type. Phe362 is shown in purple; G2. Structure of Phe362; G3. Phe362 forms H-bonds with Tyr358 and Ala366 in the wild type, predicted $\mathrm{H}$ bonds are indicated by green dashed lines. Phe362 is shown in purple. $\mathrm{H} 1-\mathrm{H} 3$. Homology modeling of mutant CHST14 variant. H1. Neighboring residues of Cys 362 in the mutant CHST14; H2. Structure of Cys362; H3. Cys362 forms H-bonds with Tyr358 and Ala366 in the mutant type. 